\title{
Urinary sodium and potassium excretion and the risk of type 2 diabetes: a prospective study in Finland
}

Received: 17 November 2004 / Accepted: 20 March 2005 / Published online: 22 June 2005

(C) Springer-Verlag 2005

\begin{abstract}
Aims/hypothesis: No previous studies on the association between salt intake and the risk of type 2 diabetes have been reported. The aim of this study was to assess whether high salt intake, measured by 24 -h urinary sodium excretion, is an independent risk factor for type 2 diabetes. Methods: We followed prospectively 932 Finnish men and 1,003 women aged 35-64 years with complete data on 24-h urinary sodium and potassium excretion and other study parameters. Hazard ratios for the incidence of type 2 diabetes were estimated for different levels of 24-h urinary sodium and potassium excretion. Results: During a mean follow-up of 18.1 years, there were 129 incident cases of type 2 diabetes. The multivariate-adjusted (age, sex, study year, body mass index, physical activity, systolic blood pressure, antihypertensive drug treatment, education, smoking and coffee, alcohol, fruit, vegetable, sausage, bread and saturated fat consumption) hazard ratio for diabetes for the highest vs combined lower quartiles of 24-h urinary sodium excretion was 2.05 (95\% CI, 1.432.96). This positive association persisted in non-obese and obese subjects, in normotensive and hypertensive subjects, as well as in men and women. Potassium excretion was not associated with the risk of type 2 diabetes. Conclusions/
\end{abstract}

\footnotetext{
G. Hu $(\bowtie) \cdot$ P. Jousilahti · M. Peltonen · J. Lindström •

J. Tuomilehto

Diabetes and Genetic Epidemiology Unit,

Department of Epidemiology and Health Promotion,

National Public Health Institute,

Mannerheimintie 166,

00300 Helsinki, Finland

e-mail: hu.gang@ktl.fi

Tel.: +358-9-19127366

Fax: +358-9-19127313

G. Hu $\cdot$ P. Jousilahti $\cdot$ M. Peltonen $\cdot$ J. Lindström ·

J. Tuomilehto

Department of Public Health,

University of Helsinki,

Helsinki, Finland

J. Tuomilehto

South Ostrobothnia Central Hospital,

Seinäjoki, Finland
}

interpretation: High sodium intake predicted the risk of type 2 diabetes, independently of other risk factors including physical inactivity, obesity and hypertension. These results provide direct evidence of the harmful effects of high salt intake in the adult population, although the confounding effect of other dietary factors cannot be fully excluded.

Keywords Risk factor - Salt intake - Type 2 diabetes Urinary potassium $\cdot$ Urinary sodium

Abbreviations MONICA: MONItoring trends and determinants of CArdiovascular disease - WHO: World Health Organization

\section{Introduction}

Type 2 diabetes is one of the fastest growing public health problems in developed and developing countries [1]. Hypertension is extremely common in patients with type 2 diabetes, affecting up to $60 \%$ of diabetic patients [2]. Furthermore, the results from epidemiological studies have indicated that subjects with hypertension are more likely to develop type 2 diabetes [3]. Thus, hypertension and diabetes may have some common underlying relationship and coexistent risk factors. Sedentary lifestyle, obesity and ageing are the common risk factors for both hypertension and type 2 diabetes [4-7]. Several dietary factors have also been associated with insulin sensitivity, glucose metabolism and risk of hypertension and type 2 diabetes $[8,9]$.

High intake of dietary sodium, low intake of potassium and high urinary sodium to potassium ratio have repeatedly demonstrated a direct relationship with essential hypertension [10-15]. Some studies [16-18] but not all [19] have demonstrated that hyperinsulinaemia and insulin resistance were present in normotensive or hypertensive salt-sensitive patients compared with salt-resistant subjects. Higher intake of magnesium might improve glucose disposal and insulin action and reduce the risk of the development of type 2 diabetes $[20,21]$. However, dietary magnesium in- 
take seems to be associated with sodium- and potassiumintake levels and sodium opposes magnesium and potassium in many physiological processes $[8,10,22]$. The potential association between sodium intake and the risk of type 2 diabetes has not been investigated. The aim of this study was to find out whether high sodium intake, measured by 24-h urinary sodium excretion, increased the risk of type 2 diabetes in the adult Finnish population.

\section{Subjects, materials and methods}

Participants Baseline surveys were carried out in two eastern Finnish provinces, North Karelia and Kuopio, and in the Turku-Loimaa region in south-western Finland in 1982 and 1987 [23-26]. In both surveys, the sample included men and women aged 25-64 years. The 1982 and 1987 cohorts were combined in the analyses. The original random sample was divided by area, sex and 10-year age group according to the World Health Organization (WHO) MONItoring trends and determinants of CArdiovascular disease (MONICA) protocol $[27,28]$ and comprised 3,607 subjects, of whom 2,833 (78.5\%) collected their 24-h urine specimens. An additional 301 subjects reported that they had missed some of the urine during the day of the collection and were thus excluded from the present analyses. Since only nine cases of type 2 diabetes were diagnosed in participants aged 25-34 years during the follow-up, we excluded this age group. Thus, the final sample comprised 932 men and 1,003 women, after also excluding subjects with known diabetes at baseline $(n=60)$, subjects who had type 1 diabetes $(n=5)$ at baseline or during the follow-up and subjects with incomplete data on any variables required for this analysis $(n=9)$. The participants gave verbal informed consent. These surveys were conducted according to the ethical rules of the National Public Health Institute and the investigations were carried out in accordance with the Declaration of Helsinki.

Baseline measurements A self-administered questionnaire was sent to the participants to be completed at home. The questionnaire included questions on medical history, drug treatment, socioeconomic factors, smoking habits, physical activity and dietary habits. Education level, measured as the total number of school years, was divided into birth cohort-specific tertiles. Based on the responses, the participants were classified as never smoked, ex-smokers and current smokers. Current smokers were categorised into those who smoked $<20$ or $\geq 20$ cigarettes per day. Physical activity included occupational, commuting and leisuretime physical activity. A detailed description of the questions is presented elsewhere [4-7, 29-31] and these questions were similar to those used and validated in the Seven Countries study [32]. The subjects reported their occupational physical activity according to the following three categories: light, moderate or active. The daily journey to and from work was grouped into three categories: using motorised transportation or not working outside home $(0$ min of walking or cycling); walking or bicycling 1-29 min; or walking or bicycling for more than 30 min. Self-reported leisure-time physical activity was classified into three categories: low, moderate or high. Since our previous study has shown that moderate and high occupational, commuting or leisure-time physical activity independently and significantly reduced risk of type 2 diabetes [4], the three physical activity indicators were merged and regrouped into three categories: (1) low: defined as subjects who reported light levels of occupational, commuting $(<30 \mathrm{~min} /$ day) and leisure-time physical activity; (2) moderate: defined as subjects who reported only one of the three types of moderate to high physical activity; and (3) high: defined as subjects who reported two or three types of moderate to high physical activity.

The subjects' diet and food choices were assessed by three types of question [33]. First, the type of food usually consumed was evaluated (e.g. "What kind of milk, cooking fat and fat on bread do you usually choose?"). Second, the amount of food consumed daily was assessed (e.g. "How many glasses of milk and sour milk, slices of bread and cups of coffee do you have daily?") [34]. Moreover, the amount of saturated fat consumed daily from dairy products and bread fat was calculated. Finally, the frequency of consumption of vegetables and fruits over the last week ( $<1$ time/week, 1-2 times/week, 3-5 times/week, 6-7 times/ week) and the frequency of consumption of sausages over the past 12 months $(<1$ time/month, 1-2 times/month, 1 time/week, 2 times/week, almost daily, $>1$ time/day) were inquired. Alcohol consumption was categorised into three groups: none, $1-100$ and $>100$ g per week.

At the study site, specially trained nurses measured height, weight and blood pressure using a standardised protocol according to the WHO MONICA project [27]. Blood pressure was measured from the right arm of the subject, who was seated for $5 \mathrm{~min}$ before the measurement. The fifth phase of the Korotkoff sound was recorded as diastolic blood pressure. Blood pressure was measured twice and the mean of these two measurements was used in the analyses. Height was measured without shoes and weight was measured with light clothing. BMI was calculated as weight in kilograms divided by the square of the height in metres. Obesity was defined as a BMI $\geq 30.0 \mathrm{~kg} /$ $\mathrm{m}^{2}$. Hypertension was defined as systolic blood pressure $\geq 160 \mathrm{mmHg}$ and/or diastolic blood pressure $\geq 95 \mathrm{mmHg}$, or using antihypertensive drugs.

Participants were instructed to collect urine for $24 \mathrm{~h}$ and return it to the examination site the next day. They were not informed that the samples would be used to estimate their salt intake. Individuals were asked to report whether urine collection during the $24 \mathrm{~h}$ was incomplete, i.e. if some of the urine was not collected in the jar provided. At the survey site, the study nurses measured the volume of the urine collection and took a 100-ml aliquot into a plastic bottle that was sent to the central laboratory where it was stored frozen for a few months. The samples were thawed for the measurement of sodium and potassium concentration with a Technicon autoanalyser. The 24-h urinary sodium and potassium excretion values were computed from these data and the recorded volume. All samples were 
analysed in the same laboratory at the National Public Health Institute in Helsinki.

Diagnosis of diabetes We ascertained incident cases of diabetes from the National Hospital Discharge Register and the National Social Insurance Institution's Drug Register. These register data were linked to the risk factor survey data with the unique personal identification numbers assigned to every resident of Finland. Antidiabetic drugs prescribed by a physician are free of charge in Finland subject to approval of the application to the Social Insurance Institution with a case history prepared by the treating physician attached. The physician confirms the diagnosis of diabetes on the basis of the World Health Organization criteria $[35,36]$. All patients receiving freeof-charge medication (either oral antidiabetic agents or insulin) are entered into a register maintained by the Social Insurance Institution. The National Hospital Discharge Register includes hospitalisations for patients admitted to hospitals with a primary or secondary diagnosis of diabetes in Finland nationwide. Follow-up of each participant in our present analysis continued through 31 December 2002 , or until death. In addition to antidiabetic drug treatment, data on the initiation of antihypertensive drug treatment during the follow-up were also received from the records of the Social Insurance Institution's register.

Statistical analyses The association between 24-h urinary sodium and potassium excretion and the risk of type 2 diabetes was analysed using the Cox proportional hazards models. The subjects were classified according to sex- and study year-specific quartile of the 24-h urinary sodium and potassium excretion. All analyses were adjusted first for age, sex and study year and then further also for education, physical activity, smoking, coffee and alcohol consump- tion, frequency of vegetable, fruit and sausage consumption, bread consumption, saturated fat consumption, systolic blood pressure and BMI at baseline and antihypertensive drug treatment at baseline and during follow-up. The significance of the trend over different quartiles of 24-h urinary sodium and potassium excretion was tested in the same models by giving an ordinal numeric value for each dummy variable. Since none of the interactions between sex and the 24-h urinary sodium and potassium excretion on the risk of diabetes were statistically significant, men and women were combined in the analyses. Statistical significance was considered to be $p<0.05$. All statistical analyses were performed with SPSS for Windows 12.0 (SPSS, Chicago, IL).

\section{Results}

A total of 129 cases of type 2 diabetes were identified during a mean follow-up period of 18.1 years. Age, systolic blood pressure, the prevalence of low physical activity and the consumption of alcohol, bread, fruits, vegetables and saturated fat did not differ between the quartiles of the 24-h sodium excretion (Table 1). There was a significantly increasing trend in the mean values of 24-h potassium excretion, BMI, diastolic blood pressure and daily coffee consumption and in the prevalence of obesity and hypertension by increasing sodium intake.

Table 2 shows hazard ratios for the diabetes incidence according to the quartiles of the 24-h urinary sodium and potassium excretion, with the second quartile as a reference. The risk of type 2 diabetes was significantly higher in the highest quartile of 24-h urinary sodium excretion. Slight but not significantly increased risk was also found among the subjects in the lowest quartile of urinary sodium excre-
Table 1 Baseline characteristics by $24-\mathrm{h}$ urinary sodium excretion $^{\mathrm{a}}$

\footnotetext{
${ }^{\mathrm{a}}$ Values represent means $\pm \mathrm{SD}$ or percentages unless otherwise indicated

${ }^{\mathrm{b}}$ Cutpoints for quartiles of 24-h urinary sodium excretion were $165,212,270 \mathrm{mmol} / 24 \mathrm{~h}$ in men in 1982, 152, 202, 253 $\mathrm{mmol} / 24 \mathrm{~h}$ in men in 1987,122 , $159,200 \mathrm{mmol} / 24 \mathrm{~h}$ in women in $1982,117,150,189 \mathrm{mmol} / 24$ $\mathrm{h}$ in women in 1987

${ }^{\mathrm{c} O b e s i t y}$ was defined as body mass index $\geq 30 \mathrm{~kg} / \mathrm{m}^{2}$

${ }^{\mathrm{d}}$ Hypertension was defined as diastolic blood pressure $\geq 95$ $\mathrm{mmHg}$ and/or systolic blood pressure $\geq 160 \mathrm{mmHg}$, or using antihypertensive drugs
}

\begin{tabular}{llllll}
\hline & \multicolumn{6}{l}{ Quartile of 24-h urinary sodium excretion } & \multirow{2}{*}{$p$ value } \\
\cline { 2 - 5 } & 1 & 2 & 3 & 4 & \\
\hline Number of participants & 484 & 484 & 484 & 483 & \\
Age, years & $50.3 \pm 8.7$ & $50.0 \pm 8.5$ & $49.0 \pm 8.3$ & $49.4 \pm 8.6$ & 0.06 \\
Female, \% & 51.7 & 51.9 & 51.9 & 52.0 & $>0.2$ \\
24 h urinary potassium excretion, mmol & $68 \pm 23$ & $79 \pm 23$ & $84 \pm 23$ & $94 \pm 28$ & $<0.001$ \\
Body mass index, kg/m ${ }^{2}$ & $25.7 \pm 3.7$ & $26.5 \pm 3.6$ & $27.1 \pm 4.2$ & $28.6 \pm 4.5$ & $<0.001$ \\
Obesity ${ }^{\text {c } \%}$ & 12.8 & 16.3 & 21.1 & 32.7 & $<0.001$ \\
Systolic blood pressure, mmHg & $144 \pm 21$ & $143 \pm 19$ & $144 \pm 21$ & $145 \pm 20$ & $>0.2$ \\
Diastolic blood pressure, mmHg & $86 \pm 12$ & $86 \pm 11$ & $87 \pm 11$ & $88 \pm 11$ & 0.002 \\
Hypertension , \% & 36.0 & 36.4 & 35.1 & 44.7 & 0.006 \\
Education, years & $9.1 \pm 3.6$ & $9.0 \pm 3.7$ & $8.6 \pm 3.2$ & $8.5 \pm 3.1$ & 0.008 \\
Coffee consumption, cups/day & $4.9 \pm 2.8$ & $4.9 \pm 2.5$ & $5.2 \pm 2.6$ & $5.3 \pm 2.8$ & 0.02 \\
Alcohol consumption, g/week & $29.7 \pm 72.9$ & $29.4 \pm 58.0$ & $30.4 \pm 57.9$ & $33.9 \pm 67.8$ & $>0.2$ \\
Bread consumption, slices/day & $5.3 \pm 2.6$ & $5.5 \pm 2.7$ & $5.6 \pm 2.7$ & $5.6 \pm 2.5$ & $>0.2$ \\
Saturated fat, g/day & $21.7 \pm 19.2$ & $21.6 \pm 20.1$ & $21.9 \pm 19.0$ & $22.5 \pm 20.0$ & $>0.2$ \\
Daily consumption of vegetables, \% & 20.5 & 19.8 & 16.1 & 15.1 & 0.07 \\
Daily consumption of fruits, \% & 29.3 & 28.7 & 27.7 & 22.4 & 0.06 \\
Almost daily consumption of sausage, \% & 35.1 & 35.5 & 29.1 & 31.1 & 0.09 \\
Low physical activity, \% & 13.8 & 12.2 & 12.6 & 12.4 & $>0.2$ \\
Current smoking, \% & 19.4 & 26.4 & 20.5 & 24.8 & 0.02 \\
\hline
\end{tabular}


tion. These associations did not change appreciably after adjustment for education, physical activity, smoking, coffee and alcohol consumption, dietary variables, antihypertensive drug treatment at baseline and during follow-up and BMI. No significant association between the 24-h urinary potassium excretion and the risk of type 2 diabetes was found.

Since there was not any significant difference for the risk of type 2 diabetes within quartiles 1-3 of the 24-h urinary sodium excretion, we combined quartiles $1-3$ of the $24-\mathrm{h}$ urinary sodium excretion in the next analyses (Table 3 ). The age-, sex- and study year-adjusted hazard ratio of diabetes was $2.84(95 \% \mathrm{CI}, 2.01-4.02)$ in the highest quartile vs combined lower quartiles for the 24-h urinary sodium excretion. The relative risk of type 2 diabetes in the highest quartile of the 24-h urinary sodium excretion increased slightly after adjustment for education, physical activity, smoking, coffee and alcohol consumption and dietary variables, and it attenuated slightly after further adjustment for systolic blood pressure, antihypertensive drug treatment at baseline and during follow-up. The hazard ratio remained highly significant $(2.05$ [95\% CI, 1.43-2.96]) also after additional adjustment for BMI. When we used diastolic blood pressure instead of systolic blood pressure in the multivariate model, the hazard ratio of diabetes was 2.04 (95\% CI, 1.41-2.94). When also the age group 25-34 years was included in the analyses, the multivariate-adjusted hazard ratio was 1.91 (95\% CI, 1.34-2.71).
The observed associations were fairly similar in nonobese and obese participants, in normotensive and hypertensive patients and in men and women (Table 4). There were no significant interactions between the 24-h urinary sodium and obesity or hypertension.

\section{Discussion}

This study is the first one where the relationship between the sodium intake and the risk of type 2 diabetes has been studied prospectively based on the individual 24-h urinary sodium excretion data. High levels of sodium intake, measured by the highest quartile of the 24-h sodium excretion, significantly increased the risk of type 2 diabetes. The increase in risk was not linear but mainly observed in the highest quartile of sodium intake.

The mechanism of the association between high intake of sodium and the risk of type 2 diabetes is not completely understood. However, several putative mechanisms can be proposed. The obesity-related insulin-resistance syndrome, which describes a cluster of metabolic abnormalities (insulin resistance, hypertension, dyslipidaemia and impaired glucose regulation), has been recognised to play an important role in the development of type 2 diabetes [36-40]. A high intake of dietary sodium and the high urinary sodium to potassium ratio have been shown to have a direct relationship with essential hypertension [10-15].

Table 2 Hazard ratios of type 2 diabetes incidence according to quartiles of 24-h urinary sodium and potassium excretion

\begin{tabular}{|c|c|c|c|c|c|}
\hline & \multicolumn{4}{|c|}{ Hazard ratio ( $95 \%$ confidence interval) } & \multirow[t]{2}{*}{$p$ for trend } \\
\hline & Quartile 1 & Quartile 2 & Quartile 3 & Quartile 4 & \\
\hline \multicolumn{6}{|l|}{ 24-h sodium intake } \\
\hline Person-years & 8,820 & 8,852 & 8,852 & 8,502 & \\
\hline Model $1^{\mathrm{a}}$ & $1.38(0.78-2.44)$ & 1.00 & $1.12(0.60-2.05)$ & $3.31(2.00-5.49)$ & $<0.001$ \\
\hline Model $4^{\mathrm{d}}$ & $1.68(0.94-3.02)$ & 1.00 & $0.89(0.48-1.67)$ & $2.29(1.35-3.88)$ & $<0.001$ \\
\hline Model $5^{\mathrm{e}}$ & $1.61(0.89-2.91)$ & 1.00 & $0.85(0.45-1.58)$ & $2.24(1.32-3.79)$ & $<0.001$ \\
\hline \multicolumn{6}{|l|}{ 24-h potassium intake } \\
\hline Number of new cases & 33 & 32 & 27 & 37 & \\
\hline Person-years & 8,737 & 8,792 & 8,793 & 8,704 & \\
\hline Model $5^{\mathrm{e}}$ & $1.13(0.69-1.87)$ & 1.00 & $0.74(0.43-1.24)$ & $0.84(0.51-1.38)$ & $>0.2$ \\
\hline
\end{tabular}

\footnotetext{
${ }^{a}$ Adjusted for age, sex and study year

${ }^{\mathrm{b}}$ Adjusted for age, sex, study year, education, physical activity (low, moderate and high), smoking status (never, past and current smoking of $1-19$ or $\geq 20$ cigarettes/day), alcohol consumption $(0,1-100$ and $>100$ g/week), coffee consumption $(0-2,3-6$ and $\geq 7$ cups/day), frequency of vegetable consumption ( $<1$ time/week, 1-2 times/week, 3-5 times/week, 6-7 times/week), frequency of fruit consumption $(<1$ time/ week, 1-2 times/week, 3-5 times/week, 6-7 times/week), frequency of sausage consumption ( $<1$ time/month, 1-2 times/month, 1 time/ week, 2 times/week, almost daily, $>1$ time/day), bread consumption (slices/day) and saturated fat consumption (g/day)

${ }^{\mathrm{c}}$ Adjusted for variables in model 2 and systolic blood pressure and antihypertensive drug treatment at baseline and during follow-up

${ }^{\mathrm{d}}$ Adjusted for variables in model 2 and body mass index

eAdjusted for variables in model 2 and systolic blood pressure, antihypertensive drug treatment at baseline and during follow-up and body mass index
} 
Table 3 Hazard ratios of type 2 diabetes incidence associated with 24-h urinary sodium excretion

\begin{tabular}{ll} 
Hazard ratio (95\% confidence interval) \\
$\begin{array}{ll}\text { Quartiles } 1-3 \text { of } 24-\mathrm{h} & \text { Quartile } 4 \text { of } 24-\mathrm{h} \\
\text { sodium intake } & \text { sodium intake }\end{array}$ \\
\hline
\end{tabular}

$\begin{array}{lll}\begin{array}{l}\text { Number of new } \\ \text { cases }\end{array} & 69 & 60 \\ \text { Person years } & 26,524 & 8,502 \\ \text { Model } 1^{\mathrm{a}} & 1.00 & 2.84(2.01-4.02) \\ \text { Model } 2^{\mathrm{b}} & 1.00 & 3.01(2.11-4.28) \\ \text { Model } 3^{\mathrm{c}} & 1.00 & 2.80(1.96-4.00) \\ \text { Model } 4^{\mathrm{d}} & 1.00 & 2.05(1.43-2.96)\end{array}$

${ }^{\mathrm{a} A d j u s t e d ~ f o r ~ a g e, ~ s e x ~ a n d ~ s t u d y ~ y e a r ~}$

${ }^{\mathrm{b}}$ Adjusted for age, sex, study year, education, physical activity (low, moderate and high), smoking status (never, past and current smoking of $1-19$ or $\geq 20$ cigarettes/day), alcohol consumption $(0$, $1-100$ and $>100 \mathrm{~g} /$ week), coffee consumption $(0-2,3-6$ and $\geq 7$ cups/day), frequency of vegetable consumption ( $<1$ time/week, 1-2 times/week, 3-5 times/week, 6-7 times/week), frequency of fruit consumption ( $<1$ time/week, 1-2 times/week, 3-5 times/week, 6-7 times/week), frequency of sausage consumption $(<1$ time/month, 1-2 times/month, 1 time/week, 2 times/week, almost daily, $>1$ time/ day), bread consumption (slices/day) and saturated fat consumption (g/day)

${ }^{\mathrm{c}}$ Adjusted for variables in model 2 and systolic blood pressure and antihypertensive drug treatment at baseline and during follow-up

${ }^{\mathrm{d}}$ Adjusted for variables in model 2 and systolic blood pressure, antihypertensive drug treatment at baseline and during follow-up and body mass index

Overweight and obese people usually eat more food than normal weight people; along with a bigger quantity of food intake, they also get more sodium [13]. It could be hypothesised that high sodium intake might increase the risk of type 2 diabetes partly through weight gain and hypertension. We found, however, that high levels of the urinary 24-h sodium excretion increased the risk of type 2 diabetes, independently of obesity and hypertension.

Another mechanism could be the effect of sodium intake on serum insulin concentration or insulin resistance, but the results from experimental studies are controversial. Some studies have suggested that hyperinsulinaemia and insulin resistance were present in normotensive and hypertensive salt-sensitive patients [16-18]. These findings suggest that salt sensitivity may be associated with insulin sensitivity and that salt intake may have different effects on insulin sensitivity in salt-sensitive compared with saltresistant persons. Most studies [16-18], but not all [19], have demonstrated a relationship between insulin resistance and salt sensitivity. Another controversy exists concerning the effect of salt intake on serum insulin concentrations. Some studies have presented that a change in salt intake would lead to changes in insulin sensitivity and/or serum insulin concentration $[16,19]$. However, other analyses have reported that variation in salt intake had no effect on either insulin sensitivity or serum insulin concentration [17]. The use of different methodologies to measure both salt sensitivity and insulin sensitivity may be the main reason to explain these conflicting results. We did not measure serum insulin in our study and this is one limitation of our study.

The effect of dietary sodium intake on the risk of type 2 diabetes may also interact with the intake of other nutrients, such as magnesium, potassium, calcium and fibre. Low intake of potassium and a high urinary sodium to potassium ratio have indicated a direct relationship with essential hypertension [10-15]. A significant inverse relationship between magnesium intake and the risk of diabetes has been found in several prospective studies [20, 21]. The opposing effects of high levels of sodium and low levels of magnesium and potassium on the risk of type 2 diabetes are a potential explanation for the observed results. Higher sodium intake may be associated with an unhealthy

Table 4 Hazard ratios of type 2 diabetes incidence associated with 24-h urinary sodium excretion (the highest quartile vs quartiles 1-3) among various subpopulations

\begin{tabular}{|c|c|c|c|c|c|}
\hline & \multirow{2}{*}{$\begin{array}{l}\text { Number of new cases } \\
\text { (quartiles } 4 \text { /quartiles 1-3) }\end{array}$} & \multirow{2}{*}{$\begin{array}{l}\text { Person-years } \\
\text { (quartiles 4/quartiles 1-3) }\end{array}$} & \multicolumn{3}{|c|}{ Hazard ratio ( $95 \%$ confidence interval) } \\
\hline & & & Model $1^{\mathrm{a}}$ & Model $2^{\mathrm{b}}$ & Model $3^{\mathrm{c}}$ \\
\hline Body mass index $<30 \mathrm{~kg} / \mathrm{m}^{2}$ & $20 / 37$ & $5,897 / 22,239$ & $2.16(1.25-3.73)$ & $2.28(1.30-3.97)$ & $1.89(1.07-3.35)$ \\
\hline Body mass index $\geq 30 \mathrm{~kg} / \mathrm{m}^{2}$ & $40 / 32$ & $2,605 / 4,285$ & $2.20(1.38-3.52)$ & $2.58(1.58-4.23)$ & $2.00(1.20-3.33)$ \\
\hline Non-hypertension & $16 / 23$ & $4,807 / 17,167$ & $2.54(1.34-4.82)$ & $2.53(1.30-4.95)$ & $1.98(1.01-3.93)$ \\
\hline Hypertension $^{\mathrm{d}}$ & $44 / 46$ & $3,695 / 9,357$ & $2.61(1.72-3.96)$ & $2.87(1.86-4.41)$ & $2.11(1.36-3.29)$ \\
\hline Men & $35 / 32$ & $4,054 / 12,875$ & $3.63(2.25-5.85)$ & $4.28(2.59-7.08)$ & $2.80(1.63-4.81)$ \\
\hline Women & $25 / 37$ & $4,448 / 13,649$ & $2.18(1.31-3.62)$ & $2.15(1.27-3.65)$ & $1.49(0.86-2.58)$ \\
\hline
\end{tabular}

No interactions between 24-h urinary sodium and each of obesity, hypertension and sex on risk of diabetes

${ }^{a}$ Adjusted for age, sex (except analyses by sex) and study year

${ }^{\mathrm{b}}$ Adjusted for age, sex (except analyses by sex), study year, physical activity (low, moderate and high), smoking status (never, past and current smoking of $1-19$ or $\geq 20$ cigarettes/day), alcohol consumption $(0,1-100$ and $>100 \mathrm{~g} / \mathrm{week})$, coffee consumption (0-2, 3-6 and $\geq 7$ cups/day), frequency of vegetable consumption ( $<1$ time/week, 1-2 times/week, 3-5 times/week, 6-7 times/week), frequency of fruit consumption ( $<1$ time/week, 1-2 times/week, 3-5 times/week, 6-7 times/week), frequency of sausage consumption $(<1$ time/month, 1-2 times/month, 1 time/week, 2 times/week, almost daily, $>1$ time/day), bread consumption (slices/day) and saturated fat consumption (g/day) ${ }^{\mathrm{c}}$ Adjusted for variables in model 2 and systolic blood pressure (except in hypertensive/non-hypertensive analyses), antihypertensive drug treatment at baseline and during follow-up (except in hypertensive/non-hypertensive analyses) and body mass index

${ }^{\mathrm{d}}$ Hypertension was defined as diastolic blood pressure $\geq 95 \mathrm{mmHg}$ and/or systolic blood pressure $\geq 160 \mathrm{mmHg}$, or using antihypertensive drugs 
diet regarding other aspects of diet. A diet high in sodium increases the need to drink more. After salty snacks people often drink sweet soft drinks, which may increase the level of postprandial glucose and may also lead to obesity and imbalanced nutrient intakes due to a reduced intake of healthier foods. The data from the Health Professionals' Follow-up Study [41] and the Nurses' Health Study [42] also found that a diet high in processed meats could increase the risk for developing type 2 diabetes. A diet with a high intake of salt from processed meats may be one potential explanation of above association. We cannot fully exclude the possibility that the observed association is due to residual confounding of obesity or some measured dietary factors or confounding of some unmeasured dietary factors.

Clinical trials have shown that a healthy diet and enhanced physical activity are effective in preventing type 2 diabetes in high-risk individuals [43-45]. Our finding represents another important dietary determinant of type 2 diabetes. A particular strength of the present study is that we measured the 24-h urinary sodium excretion, which is regarded as the best way to measure the sodium intake of an individual [46], but collection of 24-h urinary samples in large population studies is very difficult. A limitation of our study was that we did not carry out a glucose tolerance test at baseline and during follow-up. Therefore, we could have missed some cases of asymptomatic and diet-treated diabetes, although the clinical diagnosis of diabetes from the hospital discharge register may have, in part, avoided this potential underdiagnosis. We cannot exclude completely either the effects of residual confounding due to measurement error in the assessment of confounding factors, or some unmeasured dietary factors (such as intake of fibre, magnesium, calcium, whole grain, glycaemic load of the diet and total energy intake) although we have included some dietary variables in the multivariate analysis.

In conclusion, this study demonstrated that a high sodium intake predicts the risk of type 2 diabetes, but the confounding effect of other dietary factors cannot be fully excluded. This novel finding needs to be confirmed in other studies and it would be important to investigate mechanisms behind the association of high sodium intake and the risk of type 2 diabetes.

Acknowledgements This study was supported by grants from the Finnish Academy (grants 46558, 53585, 204274, 205657).

\section{References}

1. King H, Aubert RE, Herman WH (1998) Global burden of diabetes, 1995-2025: prevalence, numerical estimates, and projections. Diabetes Care 21:1414-1431

2. American Diabetes Association (2003) Treatment of hypertension in adults with diabetes. Diabetes Care 26(Suppl 1):S80S82

3. Meisinger C, Thorand B, Schneider A, Stieber J, Doring A, Lowel H (2002) Sex differences in risk factors for incident type 2 diabetes mellitus: the MONICA Augsburg cohort study. Arch Intern Med 162:82-89
4. Hu G, Qiao Q, Silventoinen K et al (2003) Occupational, commuting, and leisure-time physical activity in relation to risk for type 2 diabetes in middle-aged Finnish men and women. Diabetologia 46:322-329

5. Hu G, Barengo NC, Tuomilehto J, Lakka TA, Nissinen A, Jousilahti P (2004) Relationship of physical activity and body mass index to the risk of hypertension: a prospective study in Finland. Hypertension 43:25-30

6. Hu G, Lindstrom J, Valle TT et al (2004) Physical activity, body mass index, and risk of type 2 diabetes in patients with normal or impaired glucose regulation. Arch Intern Med 164:892-896

7. Barengo NC, Hu G, Kastarinen M et al (2005) Low physical activity as a predictor for antihypertensive drug treatment in 25-64-year-old populations in Eastern and south-western Finland. J Hypertens 23:293-299

8. Srinath Reddy K, Katan MB (2004) Diet, nutrition and the prevention of hypertension and cardiovascular diseases. Public Health Nutr 7:167-186

9. Steyn NP, Mann J, Bennett PH et al (2004) Diet, nutrition and the prevention of type 2 diabetes. Public Health Nutr 7:147165

10. Intersalt Cooperative Research Group (1989) The INTERSALT study. J Hum Hypertens 3:279-331

11. Law MR, Frost CD, Wald NJ (1991) By how much does dietary salt reduction lower blood pressure? I. Analysis of observational data among populations. BMJ 302:811-815

12. Tian HG, Nan Y, Shao RC et al (1995) Associations between blood pressure and dietary intake and urinary excretion of electrolytes in a Chinese population. J Hypertens 13:49-56

13. Elliott P, Stamler J, Nichols R et al (1996) Intersalt revisited: further analyses of 24 hour sodium excretion and blood pressure within and across populations. Intersalt Cooperative Research Group. BMJ 312:1249-1253

14. Midgley JP, Matthew AG, Greenwood CM, Logan AG (1996) Effect of reduced dietary sodium on blood pressure: a metaanalysis of randomized controlled trials. JAMA 275:1590-1597

15. Hu G, Tian H (2001) A comparison of dietary and non-dietary factors of hypertension and normal blood pressure in a Chinese population. J Hum Hypertens 15:487-493

16. Sharma AM, Ruland K, Spies KP, Distler A (1991) Salt sensitivity in young normotensive subjects is associated with a hyperinsulinemic response to oral glucose. J Hypertens 9:329335

17. Sharma AM, Schorr U, Distler A (1993) Insulin resistance in young salt-sensitive normotensive subjects. Hypertension 21:273279

18. Giner V, Coca A, de la Sierra A (2001) Increased insulin resistance in salt sensitive essential hypertension. J Hum Hypertens $15: 481-485$

19. Melander O, Groop L, Hulthen UL (2000) Effect of salt on insulin sensitivity differs according to gender and degree of salt sensitivity. Hypertension 35:827-831

20. Lopez-Ridaura R, Willett WC, Rimm EB et al (2004) Magnesium intake and risk of type 2 diabetes in men and women. Diabetes Care 27:134-140

21. Song Y, Manson JE, Buring JE, Liu S (2004) Dietary magnesium intake in relation to plasma insulin levels and risk of type 2 diabetes in women. Diabetes Care 27:59-65

22. Chobanian AV, Hill M (2000) National heart, lung, and blood institute workshop on sodium and blood pressure: a critical review of current scientific evidence. Hypertension 35:858-863

23. Tuomilehto J, Puska P, Nissinen A et al (1984) Communitybased prevention of hypertension in North Karelia, Finland. Ann Clin Res 16(Suppl 43):18-27

24. Tuomilehto J, Pietinen P, Uusitalo U, Korhonen H, Nissinen A (1989) Changes in sodium and potasium intake in Finland during the 1980s. Elsevier Science B.V., Amsterdam, the Netherlands

25. Vartiainen E, Puska P, Pekkanen J, Tuomilehto J, Jousilahti P (1994) Changes in risk factors explain changes in mortality from ischaemic heart disease in Finland. BMJ 309:23-27 
26. Tuomilehto J, Jousilahti P, Rastenyte D et al (2001) Urinary sodium excretion and cardiovascular mortality in Finland: a prospective study. Lancet 357:848-851

27. WHO MONICA Project Principal Investigators (1988) The World Health Organization MONICA Project (monitoring trends and determinants in cardiovascular disease): a major international collaboration. WHO MONICA Project Principal Investigators. J Clin Epidemiol 41:105-114

28. Pajak A, Kuulasmaa K, Tuomilehto J, Ruokokoski E (1988) Geographical variation in the major risk factors of coronary heart disease in men and women aged 35-64 years. The WHO MONICA Project. World Health Stat Q 41:115-140

29. Barengo NC, Hu G, Lakka TA, Pekkarinen H, Nissinen A, Tuomilehto J (2004) Low physical activity as a predictor for total and cardiovascular disease mortality in middle-aged men and women in Finland. Eur Heart J 25:2204-2211

30. $\mathrm{Hu}$ G, Eriksson J, Barengo NC et al (2004) Occupational, commuting, and leisure-time physical activity in relation to total and cardiovascular mortality among Finnish subjects with type 2 diabetes. Circulation 110:666-673

31. Hu G, Tuomilehto J, Silventoinen K, Barengo N, Jousilahti P (2004) Joint effects of physical activity, body mass index, waist circumference and waist-to-hip ratio with the risk of cardiovascular disease among middle-aged Finnish men and women. Eur Heart J 25:2212-2219

32. Keys A (1980) Seven countries: a multivariate analysis of death and coronary heart disease. Harvard University Press, Cambridge, MA

33. Lahti-Koski M, Pietinen P, Heliovaara M, Vartiainen E (2002) Associations of body mass index and obesity with physical activity, food choices, alcohol intake, and smoking in the 19821997 FINRISK Studies. Am J Clin Nutr 75:809-817

34. Tuomilehto J, Hu G, Bidel S, Lindstrom J, Jousilahti P (2004) Coffee consumption and risk of type 2 diabetes mellitus among middle-aged Finnish men and women. JAMA 291:1213-1219

35. WHO Study Group on Diabetes Mellitus (1985) Diabetes mellitus: report of a WHO study group. WHO Technical Report Series no 727. World Health Organization, Geneva

36. WHO Consultation (1999) Definition, diagnosis and classification of diabetes mellitus and its complications: Part 1 . Diagnosis and classification of diabetes mellitus. Report of a WHO Consultation 99.2. Geneva
37. Laaksonen DE, Lakka HM, Niskanen LK, Kaplan GA, Salonen JT, Lakka TA (2002) Metabolic syndrome and development of diabetes mellitus: application and validation of recently suggested definitions of the metabolic syndrome in a prospective cohort study. Am J Epidemiol 156:1070-1077

38. Wang JJ, Hu G, Miettinen ME, Tuomilehto J (2004) The metabolic syndrome and incident diabetes: assessment of four suggested definitions of the metabolic syndrome in a Chinese population with high post-prandial glucose. Horm Metab Res 36:708-715

39. Wang JJ, Qiao Q, Miettinen ME, Lappalainen J, Hu G, Tuomilehto J (2004) The metabolic syndrome defined by factor analysis and incident type 2 diabetes in a Chinese population with high postprandial glucose. Diabetes Care 27:2429-2437

40. Wang JJ, Hu G, Lappalainen J, Miettinen ME, Qiao Q, Tuomilehto J (2005) Changes in features of the metabolic syndrome and incident impaired glucose regulation or type 2 diabetes in a Chinese population. Diabetes Care 28:448-450

41. van Dam RM, Willett WC, Rimm EB, Stampfer MJ, Hu FB (2002) Dietary fat and meat intake in relation to risk of type 2 diabetes in men. Diabetes Care 25:417-424

42. Schulze MB, Manson JE, Willett WC, Hu FB (2003) Processed meat intake and incidence of type 2 diabetes in younger and middle-aged women. Diabetologia 46:1465-1473

43. Pan X, Li G, Hu Y et al (1997) Effects of diet and exercise in preventing NIDDM in people with impaired glucose tolerance. The Da Qing IGT and diabetes study. Diabetes Care 20:537544

44. Tuomilehto J, Lindstrom J, Eriksson JG et al (2001) Prevention of type 2 diabetes mellitus by changes in lifestyle among subjects with impaired glucose tolerance. N Engl J Med 344:13431350

45. Knowler WC, Barrett-Connor E, Fowler SE et al (2002) Reduction in the incidence of type 2 diabetes with lifestyle intervention or metformin. N Engl J Med 346:393-403

46. Elliott P (1991) Observational studies of salt and blood pressure. Hypertension 17:I3-I8 